\title{
Reflexo de dilatação pupilar e a mensuração da dor no paciente anestesiado: revisão de literatura
}

\author{
Pupillary dilation reflex and pain measurement in the \\ anesthesiated patient: literature review
}

\author{
Dilatación pupilar reflejo y medición del dolor en el paciente \\ anestesiado: revisión de la literatura
}

\author{
Ana Beatriz Foleto Brait ${ }^{1}$, Bruna Alvim Reis ${ }^{1}$, Bruno Denardi Lemos², \\ Isabela de Jesus Fernandes ${ }^{1}$, Bruno Ambrósio da Rocha ${ }^{3}$, \\ Mirto Nelso Prandini ${ }^{4}$
}

\begin{abstract}
1.Estudante Curso de Medicina UNIFAI, Adamantina-SP, Brasil.
2.Estudante Curso de Medicina FACISB, Barretos-SP, Brasil.

3.Professor Doutor Curso de Medicina UNIFAI, Adamantina-SP, Brasil.

4.Professor Associado Livre Docente UNIFESP, São Paulo-SP, Brasil.
\end{abstract}

\begin{abstract}
Resumo
Introdução. A validação da presença de dor em pacientes sob anestesia geral permanece um desafio. A correta mensuração da sedoanalgesia pode proporcionar o uso de doses mínimas para ser atingido, evitando-se subdosagens ou superdosagens. Estudos recentes têm correlacionado a pupilometria com o nível de dor no paciente não comunicativo. Objetivos. Por meio de uma revisão bibliográfica, procuramos correlacionar a pupilometria e o reflexo de dilatação pupilar na monitorização da analgesia, no paciente sedado e não comunicativo, sob o uso de opioides e hipnóticos, bem como comparar a pupilometria com o uso de parâmetros vitais como frequência cardíaca, uso do índice de analgesia e nocicepção e o BIS. Método. Foi realizada uma revisão bibliográfica na plataforma "PubMed", em inglês, espanhol e português, entre o período de 1996 a 2020. A busca nas bases mencionadas resultou em 38 artigos. Resultados. Verificou-se que a pupilometria apresentou dados mais confiáveis na avaliação da nocicepção quando comparada com frequência cardíaca e pressão arterial, porém ainda há dificuldade de padronização de aplicação. A pupilometria mede diversos componentes: amplitude máxima, latência, velocidade de dilatação e contração, e tamanho máximo e mínimo. Quando avaliados os sinais vitais como frequência cardíaca e pressão arterial a pupilometria tem se mostrado um melhor preditor de analgesia, tornando-se um melhor indicador para a titulação, quando utilizados remifentanil ou propofol. Conclusões. A avaliação do reflexo de dilatação pupilar permite a mensuração da reatividade do sistema nervoso autônomo ao estímulo da dor, podendo proporcionar tratamento individualizado e com maior segurança.
\end{abstract}

Unitermos. Sedoanalgesia; reflexo pupilar; pupilometria

\footnotetext{
Abstract

Introduction. The validation of the presence of pain in patients under general anesthesia remains a challenge. The correct measurement of sedoanalgesia can provide the use of minimum doses of anesthetics drugs to be achieved, avoiding underdoses or overdoses. Recent studies have also correlated pupillometry with the level of pain in non-common patients. Objectives. Through a literature review, we sought to correlate pupillometry and pupillary dilation reflex in analgesia monitoring, in sedated and non-communicative patients, under the use of opioids and hypnotics, as well as to compare pupillometry with the use of vital parameters such as heart rate, use of analgesia and nociception index and BI. Method. A literature review was carried out based on articles found on the "PubMedociation, in English, Spanish and Portuguese, between the period 1996 to 2020. The search in the databases
} 
resulted in 38 articles. Results It was found that pupillometry presented more reliable data in the assessment of nociception when compared to heart rate and blood pressure, but there are still some difficulties in standardizing its application. Pupillometry measures several components: maximum amplitude, latency, speed of dilation and contraction, and maximum and minimum size. When evaluating vital signs such as heart rate and blood pressure, pupillometry has been shown to be a better predictor of analgesia, becoming a better indicator when remifentanil or propofol was used. Conclusions. The assessment of the pupillary dilation reflex allows the anesthesiologist to measure the reactivity of the autonomic nervous system to the pain stimulus being able to provide individualized treatment and with greater security.

Keywords. Sedoanalgesia, pupillary reflex, pupillometry

\title{
Resumen
}

Introducción. La validación de la presencia de dolor en pacientes bajo anestesia general sigue siendo un desafío. La correcta medición de la sedoanalgesia puede proporcionar el uso de dosis mínimas a alcanzar, evitando infradosificaciones, o sobredosis. Estudios recientes han correlacionado la pupilometría con el nivel de dolor en pacientes inusuales. Objetivo. A través de una revisión de la literatura, se buscó correlacionar la pupilometría y el reflejo de dilatación pupilar en la monitorización de la analgesia, en pacientes sedados y no comunicativos, bajo el uso de opioides e hipnóticos, así como comparar la pupilometría con el uso de parámetros vitales como el corazón. tasa, uso del índice de analgesia y nocicepción y el BIS. Método. Se realizó una revisión de la literatura a partir de los artículos encontrados en la plataforma "PubMed", en inglés, español y portugués, entre el período de 1996 a 2020. La búsqueda en las bases de datos antes mencionadas resultó en 38 artículos. Resultados. Se encontró que la pupilometría presentó datos más confiables en la evaluación de la nocicepción en comparación con la frecuencia cardíaca y la presión arterial, pero aún existe dificultad para estandarizar su aplicación. La pupilometría mide varios componentes: máxima amplitud, latencia, velocidad de dilatación y contracción y tamaño máximo y mínimo. Al evaluar los signos vitales como la frecuencia cardíaca y la presión arterial, se ha demostrado que la pupilometría es un mejor predictor de analgesia, indicado para la titulación de remifentanilo or propofol. Conclusiones. La evaluación del reflejo de dilatación pupilar permite al anestesiólogo medir la reactividad del sistema nervioso autónomo a los estímulos dolorosos, proporcionando así un tratamiento individualizado y más seguro.

Palabras clave. Sedoanalgesia, reflejo pupilar, pupilometría

Trabalho realizado na Faculdade de Medicina UNIFAI, Adamantina-SP, Brasil.

\section{INTRODUÇÃO}

\author{
Durante qualquer procedimento cirúrgico, os
} mecanismos fisiopatológicos que envolvem o controle da nocicepção devem ser bem monitorados e estabelecidos. A ocorrência de dor no perioperatório e pós-operatório pode culminar em disfunções orgânicas, o que torna indispensável o conhecimento sobre diferentes técnicas e anestésicos a fim de reduzir a dor e aumentar a recuperação do paciente ${ }^{1,2}$. 
A avaliação da presença de dor em pacientes sob anestesia geral permanece como um desafio na prática clínica. Sabe-se que uma correta mensuração da sedoanalgesia pode proporcionar o uso de doses mínimas necessárias para atingir o alvo terapêutico, evitando subdosagens, superdosagens e/ou intoxicações. A utilização de anestésicos em quantidades insuficientes pode cursar com alterações hemodinâmicas graves como taquicardia ou hipertensão, enquanto em excesso, podem culminar em retenção urinária, prurido, náuseas, depressão respiratória e até mesmo dor crônica ${ }^{3,4}$.

Portanto, deve-se atentar às doses adequadas das medicações para cada paciente, com a necessidade de medidas mais específicas para monitorar tanto a profundidade da analgesia e da hipnose, quanto do grau de nocicepção, de maneira individualizada5.

Na tentativa de avaliar adequadamente a nocicepção, analgesia e o grau anestésico existem diversos métodos já utilizados, como os próprios sinais vitais (frequência cardíaca e pressão arterial), o índice de analgesia e nocicepção (ANI) e o índice bispectral (BIS). Sendo assim, estudos recentes têm correlacionado também a pupilometria com o nível de dor no paciente não comunicativo.

Mais recentemente tem sido estudado o índice de dor pupilar (PPI), que é um novo sistema utilizado para a mensuração pupilométrica, com o objetivo de analisar o nível de analgesia em pacientes anestesiados, tanto em adultos quanto em crianças a partir dos dois anos de idade. A base 
do PPI baseia-se no fato de que estímulos nociceptivos geram intensidades crescentes de reatividade pupilar, partindo do princípio de que pupilas que reagem a baixos estímulos equivalem a uma baixa analgesia, enquanto pupilas que não reagem a estímulos altos e sustentados equivalem a uma potente analgesia ${ }^{6}$.

A mensuração do tamanho pupilar é realizada através de um dispositivo não invasivo que realiza a mensuração do diâmetro pupilar através de uma câmera infravermelha, que reconhece e rastreia a pupila, sem contato físico com o globo ocular. Cada medida requer a manutenção da pálpebra aberta por aproximadamente cinco segundos, lançando o diâmetro pupilar de forma instantânea. O PPI apresenta uma variação de 1-10, sendo 1 um alto nível de analgesia (baixa reação pupilar) e 10 uma baixa analgesia (alta reação pupilar $)^{7}$.

Por meio de uma revisão bibliográfica, procuramos correlacionar a pupilometria e o reflexo de dilatação pupilar na monitorização da analgesia, no paciente sedado e não comunicativo, sob uso de opioides e hipnóticos, bem como comparar a pupilometria com o uso de parâmetros vitais como frequência cardíaca, uso do índice de analgesia e nocicepção e o BIS.

\section{MÉTODO}

Foi realizada uma revisão bibliográfica com bases em artigos encontrados na plataforma "PubMed", utilizando as palavras: "Concentrations of Propofol", "Pupillary Diameter", 
"Pupillary Pain Index", "nociception", "heart hate", "analgesia and nociception index" e "monitoring", isoladas e em associação, em inglês, espanhol e português, entre o período de 1996 a 2020.

Após aplicados critérios de inclusão e exclusão busca nas bases mencionadas resultou em 38 artigos apresentados do presente estudo.

\section{RESULTADOS}

Os trabalhos consultados validaram a pupilometria como controle de sedoanalgesia, obedecendo a estímulos nociceptivos tanto em pacientes acordados como sedados ${ }^{7-}$ 12 (Quadro 1).

Em relação à alteração da frequência cardíaca, tanto estímulos nociceptivos como opioides administrados podem interferir nas oscilações dos batimentos por minuto ${ }^{13}$ (Quadro 1).

Verificou-se, nesta revisão, que a pupilometria é um parâmetro mais preciso na avaliação da nocicepção quando comparado com a frequência cardíaca e pressão arterial, havendo, entretanto, dificuldades de padronização de aplicação. Em ralação ao ANI, os autores alertam sobre a importância de se atentar a possíveis fatores que podem interferir em sua mensuração, além de se considerar se o paciente se encontra acordado ou não, assim como os medicamentos em uso ${ }^{6,14-17}$ (Quadro 1). 
Quadro 1. Resultados dos artigos incluídos.

\begin{tabular}{|c|c|}
\hline Autor/Ano & Conclusão \\
\hline Sabourdin $(2017)^{7}$ & $\begin{array}{l}\text { Constatou redução significante no consumo de remifentanil } \\
\text { intraoperatório quando a pupilometria foi utilizada para orientar a } \\
\text { administração do remifentanil em comparação com a prática } \\
\text { padrão. }\end{array}$ \\
\hline Aissou $(2012)^{8}$ & $\begin{array}{l}\text { Constatou que a pupilometria pode ser uma ferramenta valiosa } \\
\text { para orientar a administração de morfina no pós-operatório } \\
\text { imediato. }\end{array}$ \\
\hline Chapman (1999)9 & $\begin{array}{l}\text { Revelou-se que a resposta da dilatação da pupila aumentou } \\
\text { significantemente conforme a intensidade do estímulo aumentou. }\end{array}$ \\
\hline Duceau $(2017)^{10}$ & $\begin{array}{l}\text { O reflexo pupilar de dilatação mostrou bons resultados para } \\
\text { monitorar o nível de analgesia com opioides de ação prolongada } \\
\text { como o alfentanil durante a anestesia e o sulfentanil no pós- } \\
\text { operatório imediato, ou ainda o fentanil em terapia intensiva. No } \\
\text { entanto, a validação de tal estratégia com opioides de longa } \\
\text { duração ainda precisa de mais estudos. }\end{array}$ \\
\hline Guglielminotti $(2015)^{11}$ & $\begin{array}{l}\text { Constatou que o monitoramento do reflexo pupilar de dilatação } \\
\text { durante a anestesia pode auxiliar o anestesiologista na tomada de } \\
\text { decisão quanto à adaptação da concentração do local de efeito dos } \\
\text { opioides. }\end{array}$ \\
\hline Heimburger $(2016)^{12}$ & $\begin{array}{l}\text { Constatou que a pupilometria é uma ferramenta não invasiva } \\
\text { capaz de explorar a função do tronco cerebral na beira do leito, } \\
\text { sendo um contribuidor útil na avaliação de resultados ruins na } \\
\text { fase inicial pós-parada cardíaca. }\end{array}$ \\
\hline Ander & $\begin{array}{l}\text { Em indivíduos adultos sob anestesia geral para colecistectomia } \\
\text { laparoscópica, as alterações no índice de variabilidade da FC de } \\
\text { alta frequência refletem alterações no equilíbrio entre nocicepção } \\
\text { e analgesia. Este índice pode ser usado no intraoperatório para } \\
\text { titular analgesia para pacientes individuais. Mais testes são } \\
\text { necessários para determinar se o uso intraoperatório do índice } \\
\text { afeta os resultados dos pacientes. }\end{array}$ \\
\hline Sabourdin $(2019)^{6}$ & $\begin{array}{l}\text { Houve diminuição significante do índice de dor pupilar (PPI) após } \\
\text { administração de alfentanil. Os resultados deste estudo piloto } \\
\text { sugerem que o PPI diminui quando o nível de analgesia aumenta. } \\
\text { A medição do PPI não foi associada a uma resposta nociceptiva } \\
\text { clínica ou hemodinâmica. Este novo índice pode fornecer } \\
\text { informações úteis para adaptar individualmente a administração } \\
\text { de opioides antes de estímulos nociceptivos sob anestesia geral. }\end{array}$ \\
\hline Boselli $(2014)^{14}$ & $\begin{array}{l}\text { A medição do índice de analgesia imediatamente antes da } \\
\text { extubação após a anestesia por inalação com remifentanil foi } \\
\text { significantemente associada à intensidade da dor na chegada à } \\
\text { sala de recuperação pós-anestésica. O desempenho do índice de } \\
\text { analgesia para a previsão da dor pós-operatória imediata é bom } \\
\text { e pode ajudar os médicos a otimizar o manejo da dor aguda. }\end{array}$ \\
\hline Charier $(2019)^{15}$ & $\begin{array}{l}\text { Nosso estudo sugere que o coeficiente de variação do diâmetro } \\
\text { pupilar pode ser uma ferramenta útil para monitorar a dor em } \\
\text { pacientes conscientes durante o período pós-operatório. }\end{array}$ \\
\hline Rommel $(2012)^{16}$ & $\begin{array}{l}\text { O resultado confirma que o Índice de analgesia e nocicepção pode } \\
\text { ser um bom indicador de mudanças parassimpáticas em situação } \\
\text { emocional. }\end{array}$ \\
\hline Turan $(2017)^{17}$ & $\begin{array}{l}\text { O Índice de analgesia nocicepção é um parâmetro valioso para } \\
\text { monitorar analgesia perioperatória e pós-operatória. Tal índice é } \\
\text { eficiente para predizer a necessidade de analgesia no pós- } \\
\text { operatório imediato e, portanto, proporciona conforto ao paciente. }\end{array}$ \\
\hline
\end{tabular}




\section{DISCUSSÃO}

Os fármacos mais utilizados e conhecidos para a sedoanalgesia são os benzodiazepínicos (midazolam) e os opióides (morfina e fentanil). O propofol e o midazolam, ambos introduzidos em ambiente de terapia intensiva em 1980, são indicados para sedação a curto prazo, ou seja, inferior a 48 horas, em pacientes sob ventilação mecânica invasiva. No que diz respeito à morfina, há recomendação de seu uso em caso de estabilidade hemodinâmica, reservandose o fentanil para casos em que há comprometimento hemodinâmico ${ }^{18-20}$.

Ainda, no intuito de promover sinergismo entre medicações, pode-se combinar o propofol, como agente hipnótico, ou midazolam, como agente sedativo, com remifentanil, analgésico potente, que possui curto tempo de eliminação ${ }^{21}$. O propofol combinado com uma infusão contínua de opioides tornou-se uma técnica padrão para anestesia 22 .

Alguns estudos referem que a combinação ideal de concentração propofol-remifentanil pode ser aquela que, ao mesmo tempo em que garante um nível adequado de inibição cortical, permite a menor concentração remifentanil possível ${ }^{23}$. Sabourdin $2017^{7}$ concluiu em seu estudo que foi possível reduzir significativamente 0 consumo de remifentanil intraoperatório quando a pupilometria foi utilizada para orientar a administração do remifentanil em comparação com a prática padrão. O autor, sugere que a redução do consumo intraoperatório de remifentanil pode se 
tornar um ponto final importante para melhorar o manejo analgésico global de pacientes cirúrgicos ${ }^{24}$.

O conhecimento dos benefícios e malefícios de cada droga utilizada na sedação torna-se relevante, pois pode afetar a resposta hemodinâmica do indivíduo, interferindo em parâmetros que são utilizados rotineiramente na prática clínica para avaliar a presença ou não de dor no paciente sedado. Entre os parâmetros usualmente utilizados podemos citar a presença de taquicardia, hipertensão, sudorese e lacrimejamento, sendo a frequência cardíaca um sinal menos fidedigno do que a pressão arterial. Todavia, ressalta-se que tais parâmetros são influenciados por diversas condições hemodinâmicas e pelas próprias drogas utilizadas no ato cirúrgico, o que pode gerar interferência na avaliação ${ }^{25}$.

Durante o procedimento cirúrgico, é comum, na prática clínica, o uso dos sinais vitais para avaliação da presença de dor e correta analgesia, como a frequência cardíaca, a pressão arterial e até mesmo o intervalo R-R demonstrado no eletrocardiograma. Tais achados são inespecíficos e, portanto, devem ter ressalvas na hora da avaliação ${ }^{26}$.

Outro modo de se avaliar o grau de analgesia do paciente é através do índice de analgesia e nocicepção (ANI), responsável por mensurar a nocicepção e antinocicepção, que são refletidas na influência da ventilação sobre a frequência cardíaca do paciente, de maneira qualitativa e quantitativa 27,28 .

O ANI se baseia no princípio de que o indivíduo sem dor apresentará um predomínio do tônus parassimpático, 
enquanto aquele com dor permanece com predomínio do sistema nervoso simpático, auxiliando na aplicação de uma boa sedoanalgesia, bem como na previsão de dor no perioperatório imediato ${ }^{29}$. Ainda, o ANI pode variar em uma escala de 0-100, onde, no geral, quando menor que 50 subentende-se que há predomínio simpático e quando maior que 50 , consequentemente há predomínio parassimpático ${ }^{16}$.

Turan et al., em seu estudo em $2017^{17}$, classificaram o ANI da seguinte maneira: 0-30 como dor intensa, 30-50 dor moderada, 50-70 confortável e 70-100 como nenhuma dor e sem necessidade de analgesia. Desta forma, em pacientes sob anestesia geral, normalmente aceita-se um ANI entre 50-7030.

Jeanne et al. concluíram que o método do ANI pode não ser tão eficaz em pacientes acordados ${ }^{31}$.

O BIS é responsável por avaliar a profundidade da anestesia através de ondas eletroencefalográficas que monitoram o estado hipnótico cerebral, logo, diferentemente do ANI, este não se relaciona com a presença da nocicepção e sim com o grau de sedação. O BIS permite de maneira confiável a titulação de hipnóticos durante uma anestesia, já que atua monitorando a atividade cortical, tornando-se, portanto, um aliado indireto na avaliação da nocicepção ${ }^{32}$.

O estudo da motilidade pupilar tem interesse clínico relevante, pois atua como um indicador objetivo da sensibilidade da retina à luz e, por consequência, do nervo óptico. A sua oscilação permanente é resultado do equilíbrio entre fluxos opostos dos sistemas nervosos simpático e 
parassimpático. Desta forma, o estudo dos movimentos da pupila permite o diagnóstico de várias doenças, entre as quais distúrbios do sono (narcolepsia), esquizofrenia (reação a fármacos), síndrome de Adie, Alzheimer, dependência de narcóticos, entre outras ${ }^{33}$.

A pupilometria é a medição do diâmetro pupilar em condições basais. A pupilografia é a análise dessas respostas. Ambos os termos são usados de forma semelhante, pois fornecem informações sobre a integridade das vias autonômicas $^{34}$. A pupilometria mede diversos componentes: amplitude máxima, latência, velocidade de dilatação e contração, e tamanho máximo e mínimo 33 .

Diversos autores já investigaram e validaram a pupilometria no controle da sedoanalgesia7,10-12. O diâmetro da pupila obedece a estímulos nociceptivos que são denominados de "reflexo pupilar de dilatação", sendo observado tanto em pacientes acordados quanto naqueles anestesiados, e sua amplitude obedece proporcionalmente a intensidade dos estímulos e inversamente a quantidade de opioides administrados ${ }^{8,9}$.

Sabourdin et al. 20177, em seu estudo prospectivo simples cego conseguiram constatar uma redução significativa no consumo intraoperatório de remifentanil, através do uso da pupilometria em comparação com as práticas padrões, além de uma redução cumulativa na dose de morfina no pós-operatório imediato.

Vinclair et al. ${ }^{35}$, Wildemeersch et al. ${ }^{36}$ conseguiram resultados que atestam a pupilometria como confiável e 
segura para avaliar a analgesia do paciente crítico e sedado, visto que uma baixa pontuação no PPI permite prever com precisão e de forma repetida a ausência de nocicepção durante a aspiração endotraqueal, tomada como um estímulo nocivo padronizado.

Uma correta titulação de opioides e hipnóticos baseadas em reflexos individuais e objetivos, proporcionaria uma administração adequada com menor sobredosagem e subdosagem. Assim, o PPI tem-se mostrado como um dispositivo de cabeceira rápido, simples e fácil de usar, que mede a estimulação nociva de forma a ajudar os anestesiologistas ${ }^{36}$.

\section{CONCLUSÃO}

Existem diferentes métodos utilizados para a avaliação da dor no paciente anestesiado, como a frequência cardíaca, o ANI e até mesmo o BIS de forma indireta. A frequência cardíaca, embora seja muito utilizada rotineiramente tem se mostrado inespecífica e subjetiva. A avaliação do reflexo de dilatação pupilar no paciente anestesiado permite ao anestesiologista a mensuração da reatividade do sistema nervoso autônomo ao estímulo da dor, podendo proporcionar tratamento individualizado e com maior segurança. No entanto, ainda são necessários mais estudos a fim de padronizar o protocolo do índice de dor pupilar. 


\section{REFERÊNCIAS}

1.Gaudillière B, Fragiadakis GK, Bruggner RV, Nicolau M, Finck R, Tingle $M$, et al. Clinical recovery from surgery correlates with singlecell immune signatures. Sci Translat Med 2014;6:255ra131. https://doi.org/10.1126/scitranslmed.3009701

2. Alazawi W, Pirmadjid N, Lahiri R, Bhattacharya S. Inflammatory and immune responses to surgery and their clinical impact. Ann Surg 2016;264:73-80. https://doi.org/10.1097/SLA.0000000000001691

3. Leal PC, Clivatti J, Garcia JBS, Sakata RK. Hiperalgesia induzida por opioides (HIO). Rev Bras Anestesiol 2010;60:643-7. https://doi.org/10.1590/S0034-70942010000600011

4. Kim SH, Stoicea N, Soghomonyan S, Bergese SD. RemifentanilAcute Opioid Tolerance and Opioid-Induced Hyperalgesia: A Systematic Review. Am J Therap 2015;22:e62-74. https://doi.org/10.1097/MJT.0000000000000019

5. Huhle R, Burghardt M, Zaunseder S, Wessel N, Koch T, Malberg H, et al. Effects of awareness and nociception on heart rate variability during general anaesthesia. Physiol Meas 2012;33:207. https://doi.org/10.1088/0967-3334/33/2/207

6. Sabourdin N, Diarra C, Wolk R, Piat V, Louvet N, Constant I. Pupillary pain index changes after a standardized bolus of alfentanil under sevoflurane anesthesia: first evaluation of a new pupillometric index to assess the level of analgesia during general anesthesia. Anest Analg 2019;128:467-74. https://doi.org/10.1213/ANE.0000000000003681

7. Sabourdin N, Barrois J, Louvet N, Rigouzzo A, Guye ML, Dadure C, et al. Pupillometry-guided intraoperative remifentanil administration versus standard practice influences opioid use: a randomized study. Anesthesiol 2017;127:284-92.

https://doi.org/10.1097/ALN.0000000000001705

8. Aissou M, Snauwaert A, Dupuis C, Atchabahian A, Aubrun F, Beaussier $M$. Objective assessment of the immediate postoperative analgesia using pupillary reflex measurement: a prospective and observational study. J Am Soc Anesthesiol 2012;116:1006-12. https://doi.org/10.1097/ALN.0b013e318251d1fb

9.Chapman CR, Oka S, Bradshaw DH, Jacobson RC, Donaldson GW. Phasic pupil dilation response to noxious stimulation in normal volunteers: relationship to brain evoked potentials and pain report. Psychophysiol 1999;36:44-52.

https://doi.org/10.1017/s0048577299970373

10.Duceau B, Baubillier M, Bouroche G, Albi-Feldzer A, Jayr C. Pupillary reflex for evaluation of thoracic paravertebral block: a prospective observational feasibility study. Anest Analg 2017;125:1342-7. https://doi.org/10.1213/ANE.0000000000002003

11.Guglielminotti J, Grillot N, Paule M, Mentré F, Servin F, Montravers $P$, et al. Prediction of movement to surgical stimulation by the pupillary dilatation reflex amplitude evoked by a standardized noxious test. Anesthesiol 2015;122:985-93.

https://doi.org/10.1097/ALN.0000000000000624

12. Heimburger D, Durand M, Gaide-Chevronnay L, Dessertaine G, Moury PH, Bouzat P, et al. Quantitative pupillometry and transcranial 
Doppler measurements in patients treated with hypothermia after cardiac arrest. Resuscitation 2016;103:88-93. https://doi.org/10.1016/j.resuscitation.2016.02.026

13. Anderson TA, Segaran JR, Toda C, Sabouri AS, De Jonckheere J. High-frequency heart rate variability index: a prospective, observational trial assessing utility as a marker for the balance between analgesia and nociception under general anesthesia. Anest Analg 2020;130:1045-53.

https://doi.org/10.1213/ANE.0000000000004180

14.Boselli E, Bouvet L, Bégou G, Dabouz R, Davidson J, Deloste JY, et al. Prediction of immediate postoperative pain using the analgesia/nociception index: a prospective observational study. Bri J Anaest 2014;112:715-21. https://doi.org/10.1093/bja/aet407

15.Charier D, Vogler MC, Zantour D, Pichot V, Martins-Baltar A, Courbon $\mathrm{M}$, et al. Assessing pain in the postoperative period: Analgesia Nociception IndexTM versus pupillometry. Bri J Anaest 2019;123:e322-7. https://doi.org/10.1016/j.bja.2018.09.031

16.Rommel D, Nandrino JL, Jeanne M, Logier R. Heart rate variability analysis as an index of emotion regulation processes: Interest of the Analgesia Nociception Index (ANI). In: Annual International Conference of the IEEE Engineering in Medicine and Biology Society 2012:3432-5. https://doi.org/10.1109/EMBC.2012.6346703

17.Turan G, Ar AY, Kuplay YY, Demiroluk O, Gazi M, Akgun N, et al. Analgesia Nociception Index for perioperative analgesia monitoring in spinal surgery. Braz J Anesthesiol 2017;67:370-5. https://doi.org/10.1016/j.bjan.2017.03.004

18.Jacobi J, Fraser GL, Coursin DB, Riker RR, Fontaine D, Wittbrodt ET, et al. Clinical practice guidelines for the sustained use of sedatives and analgesics in the critically ill adult. Crit Care Med 2002;30:119-41. https://doi.org/10.1097/00003246-200201000-00020

19.Moritz RD. Sedação e analgesia em UTI: velhos fármacos, novas tendências. Rev Bras Ter Intensiva 2005;17:52-5. http://www.amib.com.br/rbti/download/artigo 2010617173859.pdf

20.Ostermann ME, Keenan SP, Seiferling RA, Sibbald WJ. Sedation in the intensive care unit: a systematic review. Jama 2000;283:1451-9. https://doi.org/10.1001/jama.283.11.1451

21.Yildirim V, Doganci S, Bolcal C, Oz BS, Kucukarslan N, Cosar A, et al. Combination sedoanalgesia with remifentanil and propofol versus remifentanil and midazolam for elective cardioversion after coronary artery bypass grafting. Adv Ther 2007;24:662-70. https://doi.org/10.1007/BF02848791

22. Barvais L, Engelman E, Eba JM, Coussaert E, Cantraine F, Kenny GN. Effect site concentrations of remifentanil and pupil response to noxious stimulation. Bri J Anaest 2003;91:347-52. https://doi.org/10.1007/BF02848791

23.Sabourdin N, Peretout JB, Khalil E, Guye ML, Louvet N, Constant I. Influence of depth of hypnosis on pupillary reactivity to a standardized tetanic stimulus in patients under propofol-remifentanil targetcontrolled infusion: a crossover randomized pilot study. Anest Analg 2018;126:70-7. https://doi.org/10.1213/ANE.0000000000001802 
24. Weinbroum AA, Rudick V, Sorkine P, Freedman M, Geller E, Halpern P. Midazolam versus propofol for long-term sedation in the ICU: a randomized prospective comparison. Intens Care Med 1997;23:125863. https://doi.org/10.1007/s001340050495

25.Guignard B. Monitoring analgesia. Best Pract Res Clin Anaesthesiol 2006;20:161-80. https://doi.org/10.1016/j.bpa.2005.09.002

26.Pomfrett $\mathrm{CJ}$. Heart rate variability, BIS and depth of anaesthesia. BJA 1999;82:659-62. https://doi.org/10.1093/bja/82.5.659

27.Logier R, Jeanne M, Dassonneville A, Delecroix M, Tavernier B. PhysioDoloris: a monitoring device for analgesia/nociception balance evaluation using heart rate variability analysis. In: Annual International Conference of the IEEE Engineering in Medicine and Biology 2010:1194-7. https://doi.org/10.1109/IEMBS.2010.5625971

28. Delecroix M, Jeanne $M$, Keribedj A, Couturier $N$, Logier R. Automated analgesic drugs delivery guided by vagal tone evaluation: Interest of the Analgesia Nociception Index (ANI). In: 35th Annual International Conference of the IEEE Engineering in Medicine and Biology Society (EMBC) 2013:1952-5. https://doi.org/10.1109/EMBC.2013.6609910

29.Abdullayev R, Yildirim E, Celik B, Sarica LT. Analgesia Nociception Index: heart rate variability analysis of emotional status. Cureus 2019;11:e4365. https://doi.org/10.7759/cureus.4365

30.Jeanne M, Logier R, De Jonckheere J, Tavernier B. Heart rate variability during total intravenous anesthesia: effects of nociception and analgesia. Auton Neurosci 2009;147:91-6. https://doi.org/10.1016/j.autneu.2009.01.005

31.Jeanne $\mathrm{M}$, Clément $\mathrm{C}$, De Jonckheere J, Logier R, Tavernier B. Variations of the analgesia nociception index during general anaesthesia for laparoscopic abdominal surgery. J Clin Monit Comp 2012;26:289-94. https://doi.org/10.1007/s10877-012-9354-0

32. Hamblin MR. Shining light on the head: photobiomodulation for brain disorders. BBA Clin 2016;6:113-24. https://doi.org/10.1016/j.bbacli.2016.09.002

33. Dias AG. Pupilometria dinâmica: uma proposta de rastreamento da posição e tamanho da pupila humana em tempo real. Uberlândia, Universidade Federal de Uberlândia, 2014. https://repositorio.ufu.br/handle/123456789/14625

34.Leon-Sarmiento FE, Prada DG, Gutiérrez C. Pupila, pupilometría y pupilografía. Acta Neurol Colomb 2008;24:188-97. http://www.acnweb.org/acta/2008 244 188.pdf

35.Larson MD, Tayefeh F, Sessler DI, Daniel M, Noorani M. Sympathetic nervous system does not mediate reflex pupillary dilation during desflurane anesthesia. J Am Soc Anesthesiol 1996;85:748-54. https://doi.org/10.1213/ANE.0000000000004173

36.Larson MD. Mechanism of opioid-induced pupillary effects. Clin Neurophysiol 2008;119:1358-64. https://doi.org/10.21454/rjaic.7518.251.wil 